\title{
Considérations sur le poids et l'âge des zébus de boucherie du Soudan et de la Haute-Volta (A.O.F.)
}

\author{
par A. LALANNE, P. MORNET, P. CHALUMEAU et R. VALIN
}

A l'occasion d'un essai de trypano-prévention chimiotherapique des troupeaux d'exportation, nous avons été amenés à peser individuellement 220 zébus, opération au cours de laquelle l'âge fut également noté. Une expérience d'une telle envergure étant réalisée pour la première fois en A.O.F., nous avons pensé qu'il était utile d'en faire connaître les résultats. Tel est le but de cette note.

\section{I. - CONDITIONS DE L'EXPÉRIENCE}

\section{A) Animaux. - Origine et état général.}

L'expérience fut faite de janvier à mars 1951, sur trois troupeaux originaires de trois régions différentes, mais appartenant tous à la race peule et à la sous-race ou variété soudanaise, caractérisée par une taille moyenne, à robe le plus souvent claire, à format léger, par opposition à la sous-race sénégalaise de plus grande taille, plus lourde et à la sous-race nigérienne dont la taille et le format seraient intermédiaires entre les deux précédentes. La composition de ces troupeaux était la suivante :

lor troupeau : 100 zébus (castrés) constituant le truupeau

2e troupeau : 85 $\quad-\quad$ constituant le troupeau

30 troupeau : $35 \quad-\quad$, " tout venant ".

Total $: \overline{220}$

Troupean no1. - Le premier troupeau avait été spécialement constitué en vue de cette expérience. Compte tenu des conditions locales d'exploitation du bétail do boucherie, il était remarquable par son homogénéité, la quasi totalité des animaux ayant atteint l'âge adulte ( 2 de 4 ans et 6 de 5 ans seulement). Par ailleurs, tous étaient en excellent état d'embonpoint, avec une bosse bien remplie, la saison des pluies étant terminée depuis moins de trois mois, les pâturages et l'abreuvement étant encore très suffisants. Les animaux, originaires du Cercle de Tougan (HauteVolta), furent rassemblés à Tougan où la pesée fut effectuée. Is n'avaient donc effectué que de courts déplacements. On ne pouvait réunir des conditions plus favorables ni de troupeau en meilleur état. Examinés au pâturage, les bœufs nous apparurent bien en chair, mais légers et hauts sur pattes. Invités à indiquer «à l'œil \& le poids moyen, en nous basant sur des observations faites sur des troupeaux d'autres régions, nous le situàmes aux environs de 300 kilos, alors que le propriétaire et le convoyeur indiquaient 325 kilos au minimum. Nous verrons par la suite qu'il était encore plus élevé ( 345 kilos). Nous savions que le poids des animaux de mème race et de même format était variable suivant les régions et que, par estimation, les écarts pouvaient être assez marqués, principalement avec les zébus de la Haute-Volta et ceux du Cercle de Tougan en particulier. Mais nous ignorions qu'ils pouvaient atre de cette importance, n'ayant pas el auparavant l'occasion de faire des expériences dans cette région.

Troupeau nos 2 et 3 . - L $\Theta$ deuxième essai de trypanoprévention chimiothérapique fut effectué dans des conditions nettement diflérenles, sur deux truupeaux tout venant provenant l'un du Cercle de Bandiagara (85 têtes), l'autre de Niafunké
(35 têtes), à leur passage à Sikasso (poste de contrôle et de sortie du bétail exporté en Côte d'Ivoire), localité où ils étaient parvenus, respectivement, après un mois et un mois et demi de marche. Mais il faut noter que la saison était favorable et que, durant le voyage, effectué par étapes de 15 à 20 kilomètres par jour, l'alimentation et l'abreuvement furent assurés dans de bonnes conditions.

\section{B) Matériel. - Opérations.}

Le matériel de pesage du bétail est pour ainsi dire encore inexistant dans nos régions; peu d'abattoirs en sont pourvus et il est inconnu en brousse. Aussi les pesées furent-elles effectuées avec une bascule du Service de l'Élevage, des Établissements Dalimier (Liège), portée de 1.500 kilos, pourvue d'un tablier métallique de $2 \mathrm{~m}$. $20 \times 1 \mathrm{~m}$. 60, transportè à pied d'œuvre au moyen d'un camion. Pour éviter des accidents en cours de route dus aux cahots et aux secousses (l'usure des couteaux notamment), elle fut entièrement démontée chaque fois.

Par ailleurs, nous avions affaire à des animaux peu dociles, n'ayant jamais été attachés et ayant toujours vécu en brousse, en perpétuelle errance. Comme installation, nous disposions d'un simple parc en banco où le troupeau était enfermé. Il n'était pas question de le faire passer dans un couloir de forçage, les animaux étant pesés à la sortie, un à un et l'aménagement prévu à cet effet s'avéra parfaitement inutile. Aussi les bœufs furent-ils capturés l'un après l'autre, suivant la méthode peule, au moyen de deux cordes de 8 metres environ, terminées pai un noeud coulant passé autour des cornes et à un postérieur. Nous assistâmes alors à quelques « corridas » sensationnelles. L'animal ainsi immobilisé, était amené près de la bascule, couché, entravé et traîné sur le plateau. Pour éviter des blessures au contact des arêtes vives, des sacs de sable furent disposés sur l'un des côtés de la bascule.

\section{II. — RÉSULTATS}

Les résultats de l'expérience sont consignés dans les tableaux ci-après (tableaux I et II) qui permettent de construire, pour chaque troupeau :

- la courbe des variations du nombre de têtes de bétail en fonction de l'âge (tableau I, courbes 1 et 1 bis);

- la courbe des variations du poids vif moyen en fonction de l'âge (tableau I, courbes 2 et 2 bis);

- la courbe des variations du nombre de têtes de bétail en fonction du poids (courbe 3 et 3 bis).

Dans le tableau II et dans le tracé des courbes correspondantes, nous avons négligé, pour le troupeau tout venant, le seul animal ayant moins de 4 ans parce que, représentant dans ce troupeau un cas exceptionnel, il pourrait fausser les résultats de l'analyse statistique. 
TABLEAU I - AGE

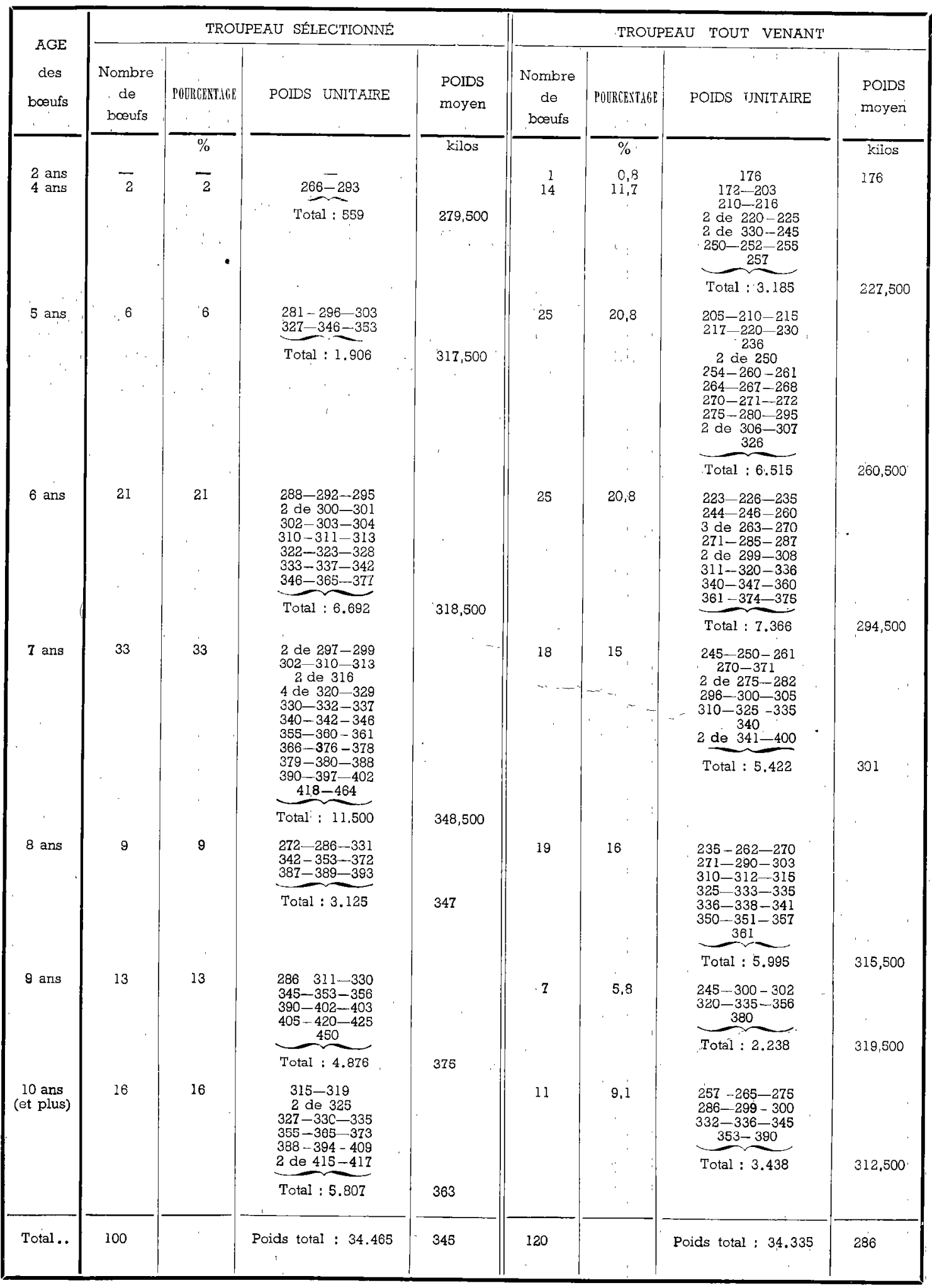


A) Les deux troupeawx $(1$ sélectionné " et " tout venant " appartiennent-ils à une mêrme popnlation d'animaux de boucherie ?

Avant tout essai d'interprétation, la première question qui se pose est celle de savoir si statistiquement les deux troupeaux peuvent être considérés comme appartenant à une même population d'animaux de boucherie (animaux de même race élevés dans les mêmes conditions de milieu, ou mieux, puisqu'il s'agit de bétail de boucherie, animaux présentant en fonction de l'âge, les variations de nombre et de poids).

La simple lecture des moyen-

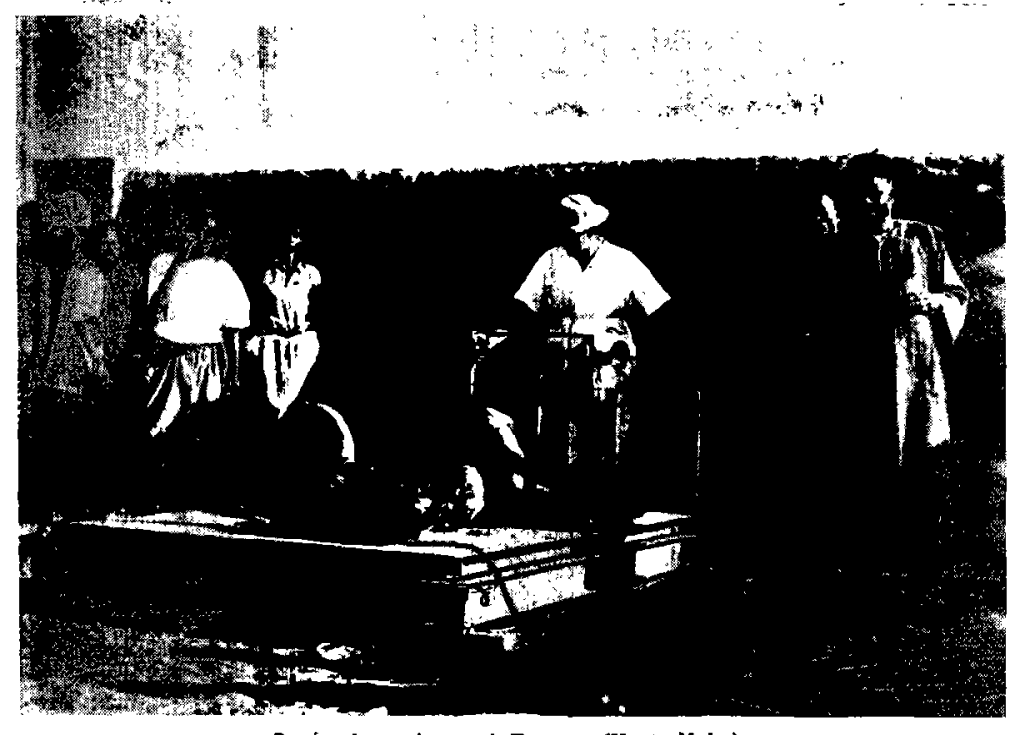
nes (en chiffres ronds 345 kilos pour le troupeau sélectionné et 287 kilos pour le troupeau tout venant) et la valeur des variantes extrêmes (260 et 480 kilos

pour le premier troupeau, 160 et 400 kilos pour le second) permettent déjà de répondre négativement. L'analyse statistique va confirmer ce résultat.

TABLEAU II

\begin{tabular}{|c|c|c|c|c|c|}
\hline \multicolumn{2}{|c|}{ POIDS EN KLOGRAMMES } & \multicolumn{2}{|c|}{ TROUPEAU SÉLECTIONNÉ } & \multicolumn{2}{|c|}{ TROUPEAU TOUT VENANT } \\
\hline Limites des classes & Centres des classes & Nombre & Pourcentage & Nombre & Pourcentay \\
\hline & & & $\%$ & & $\%$ \\
\hline $161-180$ & 170 & 一 & - & 1 & 0,84 \\
\hline $181-200$ & 190 & 一 & - & 0 & 0 \\
\hline $201-220$ & 210 & - & - & 10 & 8,40 \\
\hline $221-240$ & 230 & 一 & - & 9 & 7,56 \\
\hline $241-260$ & 250 & - & - & 16 & 13,45 \\
\hline $261-280$ & 270 & 2 & 2 & 24 & 20,16 \\
\hline $281-300$ & 290 & 13 & 13 & 13 & 10,92 \\
\hline $301-320$ & 310 & 20 & 20 & 14 & 11,77 \\
\hline $321-340$ & 330 & 18 & 18 & 14 & 11,77 \\
\hline $341-360$ & 350 & 14 & 14 & 11 & 9,25 \\
\hline $361-380$ & 370 & 11 & 11 & 5 & 4,20 \\
\hline $381-400$ & 390 & 9 & 9 & 2 & 1,68 \\
\hline $401-420$ & 410 & 10 & 10 & 一 & - \\
\hline $421-440$ & 430 & 1 & 1 & - & 一 \\
\hline $441-460$ & 450 & 1 & 1 & - & - \\
\hline $441-860$ & 470 & 1 & 1 & - & - \\
\hline \multicolumn{2}{|r|}{ Totaux............... } & 100 & 100 & 119 & 100 \\
\hline
\end{tabular}




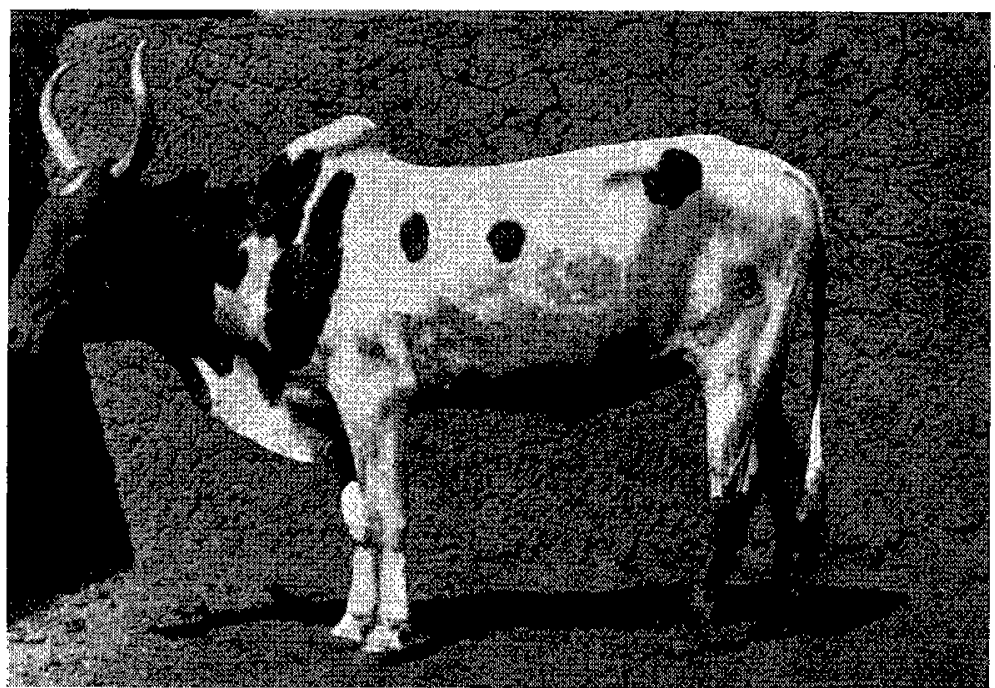

Bœeuf, 8 ans, 387 kilcs

La probabilité d'atteindre un tel écart en prenant deux troupeaux au hasard dans une même population étant pratiquement nulle, on doit les considérer comme provenant des deux populations différentes.

\section{B) Lecture des tableaux et des courbes.}

\section{Troupeau sélectionné.}

a) Les troupeaux sélectionnés en vue de l'exportation comprennent relativement peu de jeunes $(2 \%$ de 4 ans, $6 \%$ de 5 ans), un gros pourcentage de 6 ans $(21 \%)$ et de 7 ans $(33 \%)$, quelques bceufs de 8 ans $(9 \%)$, et de 9 ans $(13 \%)$ et $16 \%$ d'animaux âgés (10 ans et plus),

On connaît les effectifs $N_{1}=100$ et $N_{2}=119$ et les moyennes exactes $m_{1}=344,65$ et $m_{\mathfrak{a}}=287,05$ des deux troupeaux. Le tableau II permet d'estimer les écarts-type $S_{1}$ et $S_{2}$ des deux distributions correspondantes, d'après la formule :

$$
S^{2}=\frac{I}{N}\left(a^{2} V-\frac{a^{2} U^{2}}{N}\right)
$$

où

a représente l'intervalle de classe $(a=20)$; $N$ l'effectif du troupeau considéré;

aU la somme, étendue à toutes les classes, du produit de la fréquence par la valeur de la variable (centre des classes);

$a V$ la somme, étendue à toutes les classes, du produit de la fréquence par le carré de la valeur de la variable (cf. tableau spécial : calcul du coefficient de corrélation).

On obtient ainsi :

$$
\begin{aligned}
& \mathrm{S}_{1}=42,5 \\
& \mathrm{~S}_{2}=47,06
\end{aligned}
$$

Si les deux troupeaux étaient issus d'une même population d'animaux de boucherie, la différence des moyennes $m_{1}-m_{2}$ pour un grand nombre d'échantillons indépendants serait normalement distribuée autour d'une valeur moyenne nulle avec un écart-type de :

$$
S=\sqrt{\frac{\mathrm{S}_{1}{ }^{2}}{\mathrm{~N}_{1}}+\frac{\mathrm{S}_{2}{ }^{2}}{\mathrm{~N}_{2}}}
$$

d'où un écart réduit :

$$
t=\frac{m_{1}-m_{2}}{\mathrm{~s}}=9,5
$$

ceux-ci représentés en partie par les bceufs «conducteurs" de troupcaux, les bceufs de labour ou les bcufs porteurs dont la carrière est terminée.

Ainsi, le nombre d'animaux augmente avec l'âge jusque vers 7 ans et demi (âge moyen). Le choix à l'achat étant conditionné surtout par la taille et le format, il faudrait en conclure que l'âge adulte moyen auquel les bœufs de boucherie atteignent leur complet développement, se situe autour de 7 ans. Plus tôt, ils intéresseraient moins les acheteurs, plus tard, les ventes successives les élimineraient progressivement des troupeaux.

b) Le poids moyen augmente avec l'âge jusque vers $\theta$ ans et semble diminuer ensuite (tableau I, courbe 2), mais ces renseignements sont assez imprécis. Nous tirerons tout à l'heure des données plus sûres de la comparaison des coefficients de corrélation entre l'âge et le poids, pour la totalité des sujets : pour ceux de 4 à 9 ans, pour ceux de 4 à 8 ans, enfin, pour ceux de 4 à 7 ans.

c) Les poids présentent de grandes variations. On relève en effet :

- $15 \%$ de poids inférieurs à 300 kilos (dont $2 \%$ inférieurs à 275 kilos.

- $45 \%$ de poids variant entre 301 et 350 kilos.

- $27 \%$ de poids variant entre 351 et 400 kilos.

- $11 \%$ de poids variant entre 401 et 425 kilos.

- $2 \%$ de poids supérieurs à 425 (450 et 464 kilos).

d) Dans une même catégorie de poids, on trouve des animaux d'âges très différents, allant de 4, 5 ou 6 ans à 10 ans (et plus).

e) Dans la catégorie de poids de 450 kilos et au-dessus, on' ne trouve que 2 bœufs, l'un âgé de 
9 ans (450 kilos), l'autre de 7 ans, seulement, mais qui accuse cependant le poids le plus élevé de toute la série (464 kilos).

f) Le poids moyen est de 345 kilos.

g) Les courbes 3 et 3 bis se rapprochent des courbes en " clocher 》 des distributions normales, mais l'analyse statistique (test de conformité) révèle une différence significative avec ces dernières. Et cela se conçoit, car l'âge minimum une foix fixé (4 ans), le bétail de boucherie n'est pas acheté au hasard, mais en fonction surtout du poids et de l'état d'embonpoint.

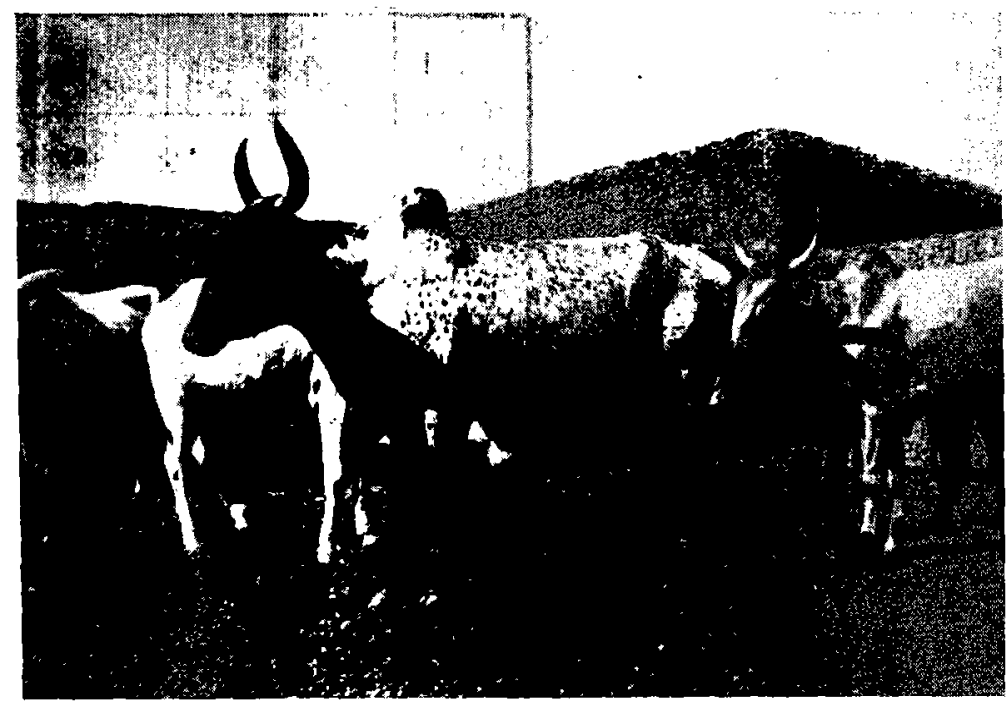

Zebu, 7 ans, 366 kilos.

\section{$2^{\circ}$ Troupeau tout venant.}

a) On trouve une proportion beaucoup plus grande d'animaux relativement jeunes. En éliminant l'unique sujet de 2 ans, on $a$, en effet, $11,8 \%$ de 4 ans, $21 \%$ de 5 ans, $21 \%$ de 6 ans, $15,1 \%$ de 7 ans, $16 \%$ de 8 ans, $5,8 \%$ de 9 ans et $9,3 \%$ de 10 ans (et plus). Ici, le nombre d'animaux augmente avec l'âge jusque vers 6 ans et demi qui représenterait l'âge adulte moyen pour les mêmes raisons que tout à l'heure. Cet âge serait inférieur à la réalité et à celui du troupeau sélectionné, le choix à l'achat, moins sévère quant à la taille et au format, laissant entrer un plus grand nombre d'animaux jeunes.

b) Le poids moyen varie avec l'âge comme précédemment, mais la moyenne de l'ensemble du troupeau est plus faible et l'amplitude de variation légèrement différente $(400-172=228$ au lieu de $464-266=198$ )

c) Les poids présentent également de grandes variations. On relève en effet :

- 60,8\% de poids inférieurs à 300 kilos (dont $49,2 \%$ inférieurs à 275 kilos, soit quatre fois plus que pour le troupeau sélectionné.

- 29,2\% de poids compris entre 301 et 350 kilos.

- $10 \%$ de poids compris entre 350 et 400 kilos (dont 2,5\% seulement supérieurs à 375 kilos).

- Pas de poids supérieurs à 400 kilos.

d) Le poids moyen augmentc avec l'âge jusque vers 9 ans et semble diminuer ensuite. L'étude des coefficients de corrélation permettra de vérifier la valeur de cette derniere indication.

e) Le poids moyen est de 286 kilos.

f) Même remarque que pour le troupeau sélectionné (alinéa g).

\section{C) Étude des coefficients de corrélation entre le poids et l'âge.}

$1^{\circ}$ Troupeau sélectionné.

a) Animaux de 4 à 10 ans (et plus).

Calcul du coefficient de corrélation (cf, tableau spécial) :

moyenne de travail :

$$
\mathrm{N}=100 \quad a-20 \quad b=1
$$

On trouve:

$$
\begin{aligned}
& x_{0}=350 \\
& y_{0}=7
\end{aligned}
$$

écart type $S x=42,5$; écart type $S y=1,58$

Coefficient de corrélation entre $x$ et $y$ :

$$
r=\frac{Z}{N S x S y}
$$

pour

$$
\mathrm{Z}=a b\left(\mathrm{~W}-\frac{\mathrm{UR}}{\mathrm{N}}\right)
$$

d'où

$$
r=0,43
$$

Ce coefficient de corrélation admet une erreur de :

d'où

$$
\frac{1-r^{2}}{\sqrt{\mathrm{N}-1}}=0,68
$$

$$
r=0,43 \pm 0,08
$$

b) Animaux de 4 à 9 ans (10 ans et plus éliminés) :

$$
\mathrm{N}=84
$$

Un calcul analogue donne :

$$
r=0,468 \pm 0,085
$$

c) Animaux de 4 à 8 ans (9 ans et plus éliminés) :

$$
\begin{aligned}
\mathrm{N} & =71 \\
r & =0,33 \pm 0,106
\end{aligned}
$$




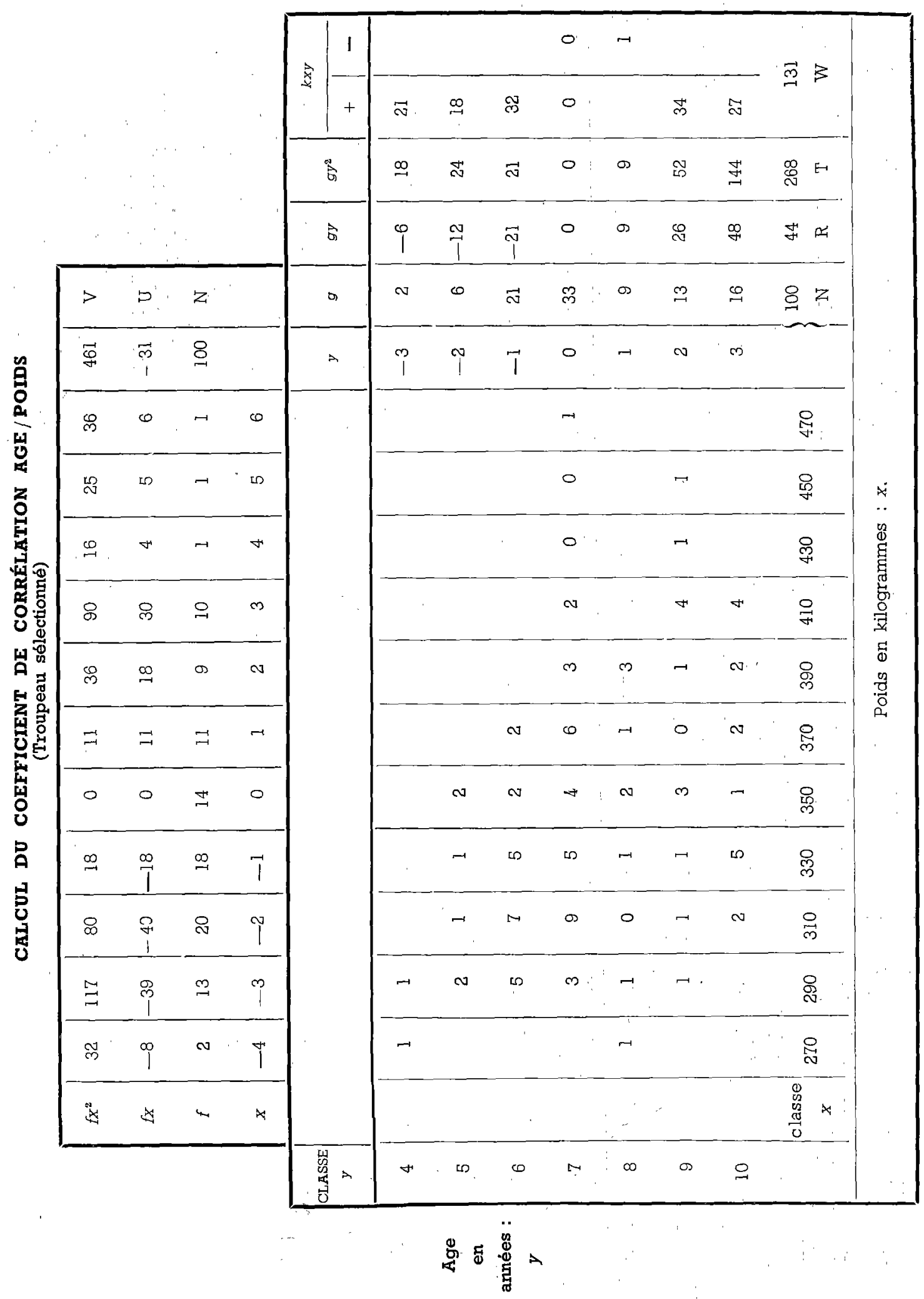


d) Animaux de 4 à 7 ans (8 ans et plus éliminés) :

$$
\begin{aligned}
\mathrm{N} & =62 \\
r & =0,35 \pm 0,112
\end{aligned}
$$

\section{$2^{\circ}$ Troupeau tout venant.}

a) Animaux de 4 à 10 ans (et plus) :

$$
\begin{aligned}
\mathrm{N} & =119 \\
r & =0,545 \pm 0,064
\end{aligned}
$$

b) Animaux de 4 à 9 ans :

$$
\begin{aligned}
\mathrm{N} & =108 \\
r & =0,592 \div 0,063
\end{aligned}
$$

c) Animaux de 4 à 8 ans :

$$
\begin{aligned}
\mathrm{N} & =101 \\
r & =0,5 \pm 0,075
\end{aligned}
$$

d) Animaux de 4 à 7 ans :

$$
\begin{aligned}
N & =82 \\
r & =0,575 \doteq 0,074
\end{aligned}
$$

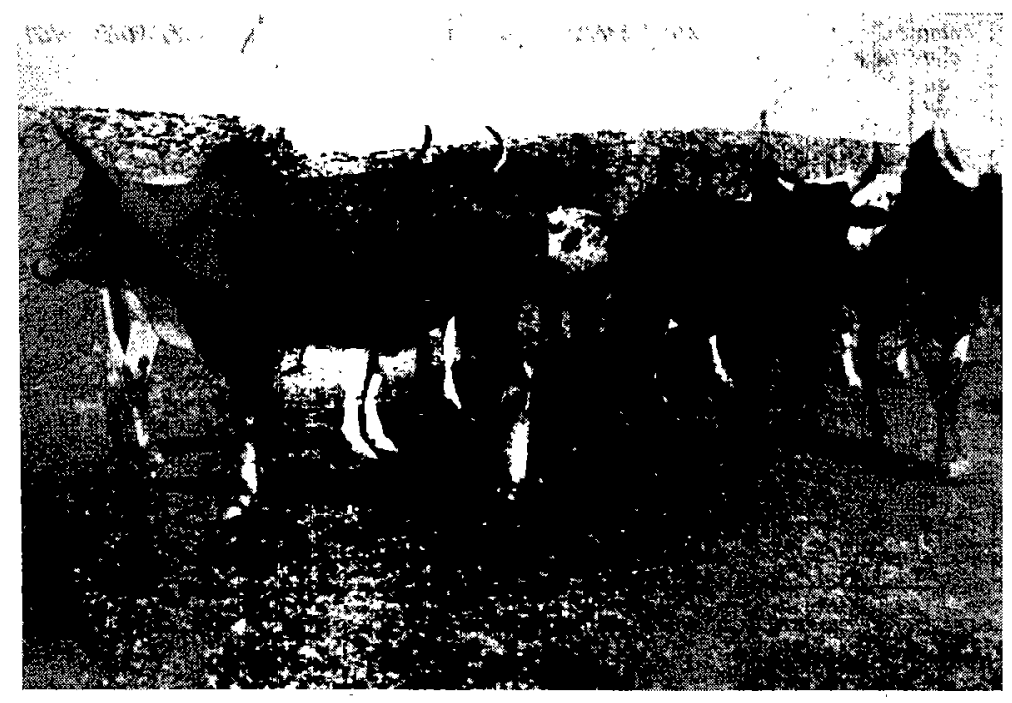

Ercellent animal de boucherie, 6 ans, 455 kilos.

$3^{\circ}$ Interprétation des résultats.

De cette étude, on peut tirer les renseignements suivants, valables pour les troupeaux en observation :

a) Il existe une corrélation positive certaine entre le poids et l'âge des bœufs de boucherie (entre 4 et 10 ans ou plus).

b) Cette corrélation apparaît ici plus forte pour le troupeau tout venant que pour le troupeau sélectionné.

c) Pour le troupeau tout venant, les variations du coefficient de corrélation après l'âge de 7 ans, sont faibles et sans signification. d) Pour le troupeau sélectionné, les variations après l'âge de 7 ans sont déjà plus fortes : de 0,35 et 0,33 pour les animaux de 1 à 7 ans et de 4 à 8 ans, le coefficient de corrélation passe à 0,468 pour ceux de 4 à 9 ans, pour retomber à 0,43 pour ceux de 4 à 10 ans et plus. Mais là encore, les différences ne sont pas significatives puisque, compte tenu de l'erreur commise sur leur estimation, le coefficient le plus bas $(0,33)$, compris entre 0,224 et 0,436 et le plus élevé $(0,468)$, entre 0,383 et 0,553 , ont un intervalle de variation commun, de 0,383 à 0,436 .

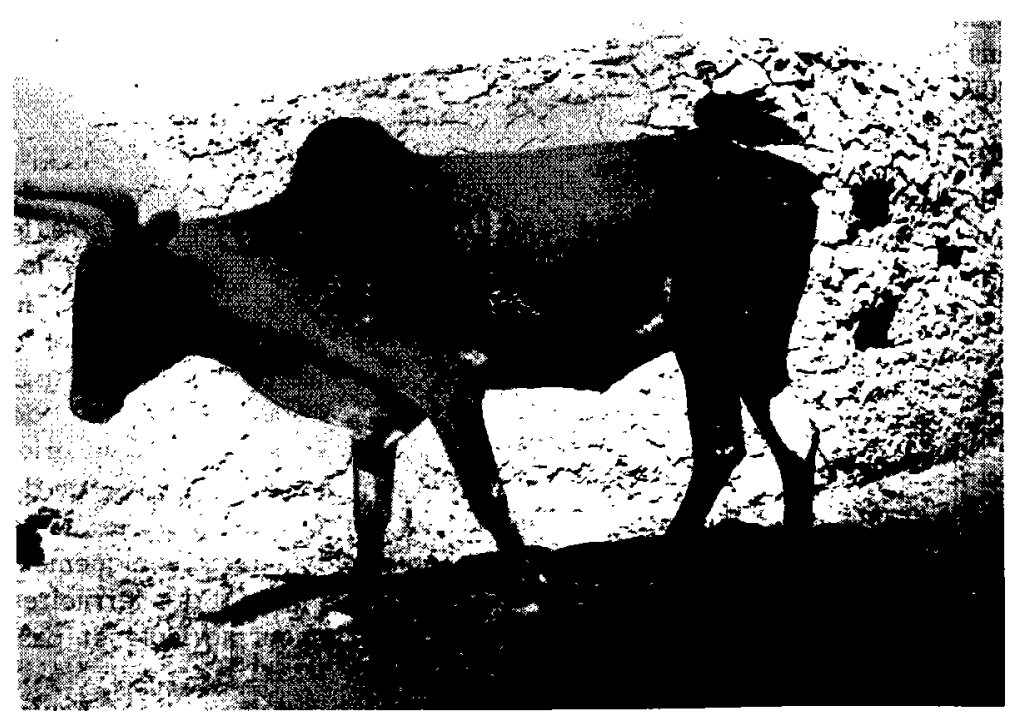

Bcuf de 9 ans, aux apparences trompeuses. 450 kilos.

\section{III. - PERTES DE POIDS EN COURS DE ROUTE}

Des distances considérables séparent les zones de production des centres de consommation, que les:bœufs de boucherie effectuent le plus souvent à pied, les chemins de fer étant rares en Afrique et les wagons à bestiaux nettement insuffisants. C'est ainsi que le troupeau sélectionné a collvert une distance de près de' 1.000 kilomètres en cinquante-neuf jours et le troupeau tout venant, environ 500 kilomètres en un mois, par étapes de 20 à 25 kilomètres par jour. Il ne faut donc point s'étonner qu'il y ait des pertes en cours de route (en nombre et 


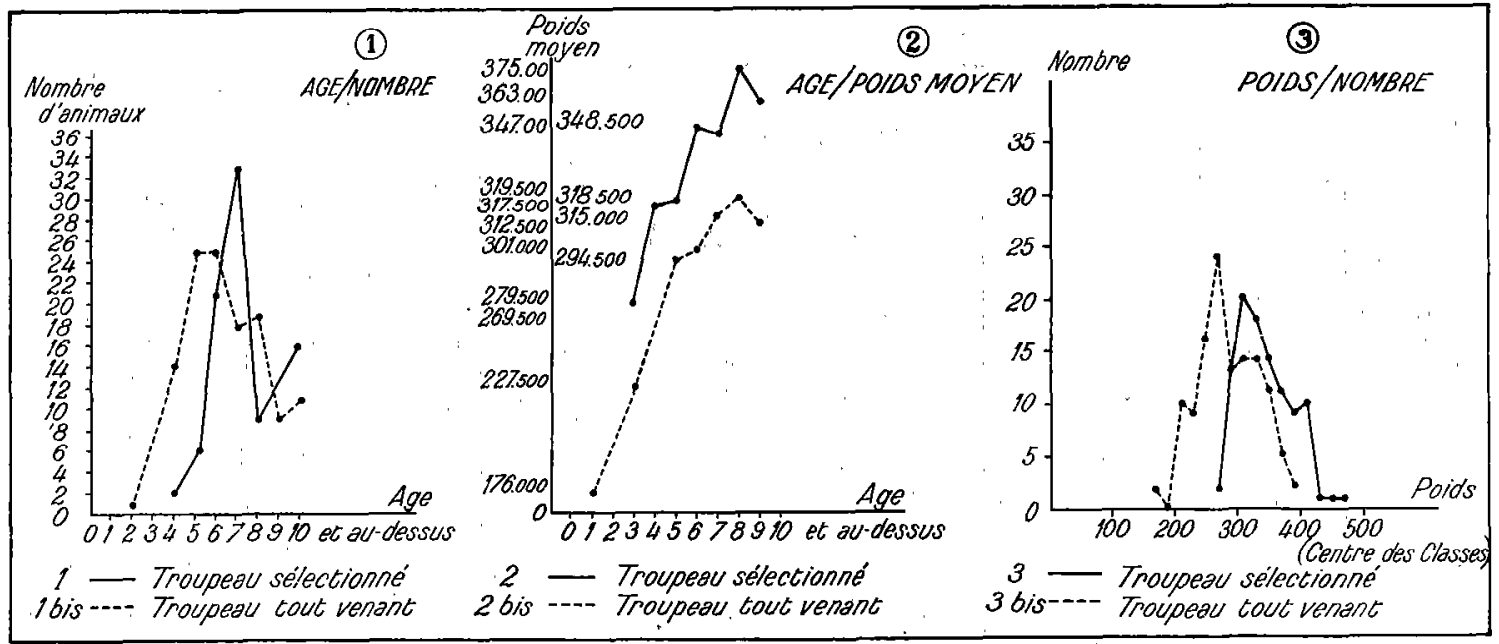

en poids), consécutives à la fatigue du voyage, à la sous-alimentation, à l'insuffisance de l'abreuvement et aux affections diverses (trypanosomiase en particulier). Nous les avons étudiées dans le détail par ailleurs ( 1 ) et nous ne mentionnerons ici que la conclusion : les pertes en poids en cours do routc dépassent parfois $20 \%$, alors que les pertes en nombre (morts, évadés, etc., sont rarement supérieures à 5, \%. Mais il convient de tenir compte également d'un nombre sensiblement équivalent de bceufs vendus en cours de route (boîteux, traînards, etc.), en général, dans de mauvaises conditions.

\section{CONCLUSIONS}

Pour les troupeaux de zébus de boucherie rentrant dans l'une des deux catégories étudiées (troupeaux 《 sélectionné » et 《 tout venant »), il se dégage de cette étude les conclusions suivantes :

$1^{\circ}$ Le bétail (zébus de boucherie) de l'A.O.F. n'est pas homogène; 'on y trouve des bœufs de tout âge et de poids variable. Mais si, dans les troupeaux. examinés, il n'y avait que des bceufs, on rencontre fréquemment des taureaux et des vaches stériles ou hors d'âge.

$2^{\circ}$ Le poids moyen est de 285 kilos pour le troupeau « tout venant 》 et de 345 kilos pour le « troupeau sélectionné » avec, respectivement, des minima de 175 à 265 kilos et des maxima de 400 à 465 kilos.

Sachant, si on avait examiné un très grand nombre de troupeaux de boucherie des deux catégories, qu'il y aurait $95 \%$ de chances pour que la vraie moyenne soit comprise entre

$$
m-\frac{2 S}{\sqrt{\bar{N}}} \text { et } m+\frac{2 S}{\sqrt{N}}
$$

on peut écrire qu'il y a $95 \%$ de chances pour que les vraies moyennes en kilos soient comprises :

(1) MORNET (P.), LALANNE (A.) et SISSOKO (M.), - Essai de trypano-prévention chimiothérapique des troupeaux bovins d'exportation (à paraître). pour le troupeau sélectionné, entre 336,5 ef 353,5 , pour le troupeau tout venant, entre 278,3 et 295,7 . 30 L'âge moyen varie entre 6 ans et demi et 7 ans et demi, les variantes extrêmes étant 4 ans (exceptionnellement 2 ans) et 10 ans et même plus.

$1^{\circ}$ Le poids moyon augmonte avoc l'âge. A partir de 7 ans cependant, la relation, entre ces deux facteurs est difficile à préciser sur des effectifs aussi faibles. Il est vraisemblable que la courbe, pour de gros effectifs, comporterait un sommet entre 7 et 9 ans pour redescendre ensuite, l'âge du développement complet variant entre 6 ans et demi et 7 ans et demi. A ce titre, il n'est pas économique de garder les animaux au-delà de 9 ans, l'âge optimum de vente se situant près du sommet probable de la courbe, entre 7 et 9 ans. Mais il faudra faire preuve de beaucoup de patience et de persévérance pour faire admettre cette donnée aux pasteurs nomades, les Peuls en particulier, en raison de leur mode de vie, de leur fatalisme, de leurs préjugés et de leurs coutumes.

$5^{\circ}$ Les centres de consommation étant très éloignés des centres de production, des mesures doivent être prises pour exploiter plus rationnellement le cheptel d'A.O.F., en raison des pertes considérables de viande au cours d'interminables déplacements le long des routes d'évacuation du bétail: Seuls les abattoir's industriels, doublés de frigorifiques, au voisinage des zones de production du cheptel et le transport de la viande sous le régime du froid par chemin de fer ou aéro-cargos, permettront de tirer un meilleur parti des animaux de boucherie, d'obtenir une viande de meilleure qualité et de récupérer tous, les sous-produits. C'est la seule façon d'exploiter rationnellement et économiquement le cheptel de l'A.O.F. dont la plupart des territoires ont une vocation essentiellement agricole et pastorale.

(Services de l'Élevage du Soudan et de la HauteVolta. Laboratoire Central de l'Elevage, Dakar). 